\title{
Single Molecule Electron Transfer Dynamics in Complex Environments
}

\author{
Vitor B. P. Leite, ${ }^{1, *}$ Luciana C.P. Alonso, ${ }^{1}$ Marshall Newton, ${ }^{2}$ and Jin Wang ${ }^{3, \dagger}$ \\ ${ }^{1}$ Departamento de Física, IBILCE, Universidade Estadual Paulista, São José do Rio Preto, SP 15054-000, Brazil \\ ${ }^{2}$ Department of Chemistry, Brookhaven National Laboratory, Upton, New York 11973-5000, USA \\ ${ }^{3}$ Department of Chemistry and Department of Physics, State University of New York at Stony Brook,
} Stony Brook, New York 11794-3400, USA

(Received 8 June 2005; published 8 September 2005)

\begin{abstract}
We propose a new theoretical approach to study the kinetics of the electron transfer (ET) under the dynamical influence of the complex environments with the first passage times (FPT) of the reaction events. By measuring the mean and high order moments of FPT and their ratios, the full kinetics of ET, especially the dynamical transitions across different temperature zones, is revealed. The potential applications of the current results to single molecule electron transfer are discussed.
\end{abstract}

DOI: 10.1103/PhysRevLett.95.118301

PACS numbers: 82.30.Fi, 82.20.Wt, 82.37.Np

Electron transfer is very important in governing many natural and biological processes, for instance, long range electron transfer in the photosynthetic reaction centers, the adenosine triphosphate (ATP) energy pump, etc. Environment has a great impact on the electron transfer dynamics. Marcus's theory [1] of electron transfer (ET) describes the influence of environments in electron transfer reactions by a single collective reaction coordinate. The classical (nonquantum) model should be a reasonable approximation provided the high frequency modes do not contribute strongly to the reaction coordinate. The free energy profile on this one-dimensional coordinate appears to be smooth. In general, the environment can be best described by a multidimensional landscape with a large number of energy valleys and therefore is intrinsically complex. The treatment of the reaction coordinate in an explicit way often becomes very difficult due to the large number of conformational degrees of freedom involved, such as the positions and the orientations of each solvent molecules (solvent coordinates). It is thus important to ask when the single collective coordinate description of Marcus breaks down and a general multidimensional description of the landscape becomes necessary [2].

This question has first been addressed by Onuchic and Wolynes (OW) [3]. They considered environments as polar solvents or polar groups in proteins (the orientations of solvent molecules are mimicked by spins) interacting with a charged cavity, representing the donor or acceptor site for electron transfer. For every temperature, this model has two thermodynamic regions in polarization space: a glassy trappinglike region with multidimensional valleys of the underlying solvent landscape, and another one exhibiting normal smooth landscape where the one-dimensional effective coordinate picture of Marcus is recovered. Further realistic studies addressing the outer shells for different layers of solvents are given by Tanimura, Leite, and Onuchic [4] with trapping transitions found in each corresponding layer. Onuchic and Wolynes [3] studied the polarization-dependent thermodynamic phase transition.
Leite and Onuchic [5,6] showed that the reactive region has a slow average dynamics below the transition temperatures; they argued that this phase transition should influence the dynamics of the system.

Electron transfer process can be seen as motions on, and transitions between, a multidimensional potential energy landscape. The landscape represents the potential energy of the system (electronic donor and acceptor states) as a function of reaction coordinates, as shown in Fig. 1. For a smooth landscape, a single coordinate description is appropriate, and for a rugged landscape, the multidimensional description is necessary. In this Letter, we give a quantitative description of this environment. In particular, we study the single molecule electron transfer reaction kinetics under influence of such dynamic environments at different temperatures.

We find that the kinetics experiences a transition from the high temperature self-averaging exponential behavior to low temperature non-self-averaging and nonexponential behavior. At even lower temperatures, we find the kinetics is single exponential again. The fluctuations of the kinetics reflect the complex structure of the underlying energy landscape. In the bulk, these fluctuations are suppressed by the large number of molecules and cannot be observed. In single molecules, in principle, one can explicitly measure these fluctuations and the corresponding statistics
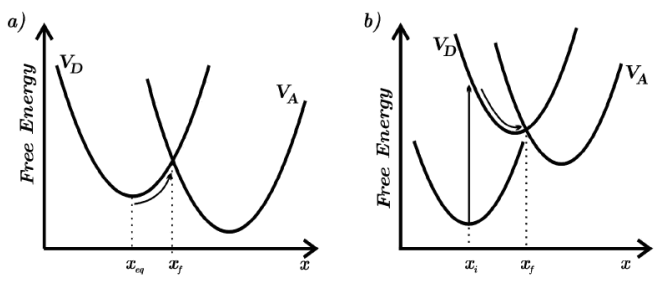

FIG. 1. Donor (D) and acceptor (A) energy surfaces for a nonadiabatic reactions: (a) The system diffuses from equilibrium to an activation polarization. (b) ET occurs after electronic excitation and solvent relaxation. 
[7,8]. To model the complex environment, we consider a shell of solvent molecules with simple rotational dynamics around a charge cavity, the OW model [3]. The solvent molecules are represented by dipoles pointing only in two directions for simplicity, as Ising spins; see Fig. 2. The total polarization $x$ per dipole can be used as the reaction coordinate for the solvent, it is defined by $x=\left(n_{+}-n_{-}\right) / N$, where $N$ is the total number of dipoles, $n_{+}\left(n_{-}\right)$is the number of dipoles oriented inward (outward) of the charge cavity, and they satisfy the condition $n_{+}+n_{-}=N$.

The interactions among dipoles and charges of the environments are heterogeneous in distances and orientations; therefore the solvent energies can be evaluated using the random energy model approximation given in [3-6,9]. The energy probability distribution $g(x, E)$ at polarization $x$ naturally follows a Gaussian distribution and is given by $g(x, E)=\frac{1}{\sqrt{2 \pi} \Delta E} \exp \left[-\frac{[E-\bar{E}(x)]^{2}}{2 \Delta E^{2}}\right]$, where $\bar{E}(x)$ is the average energy as a function of $x$ and $\Delta E$ is the variance of the energy. $\Delta E^{2}$ is assumed to be independent of $x$, and scale with the total number of dipoles $N$.

From microcanonical ensemble, we can count the number of configurational states at a given energy as a function of $x$ with energy $E$ and obtain the configuration entropy $S$. The free energy depending parametrically on the total polarization is used as the effective potential. One can obtain the transition temperature of the model, using the thermodynamic Maxwell relationship, $\partial S / \partial E=1 / T$, evaluated at the average energy at which one observes the entropic crises ( $S=0$, in the thermodynamic limit) $[3,5,6,9]$ where the system is trapped into the local minimum.

We address two transition temperatures of the OW model [3,5]: (a) Local phase transition is a kinetic transition that occurs when the system starts to get trapped in local minima. It is expected that there is a spread in the distribution of escape times from local minima. Below this temperature $\left(T_{\text {loc }}\right)$, escaping from local minima starts to be dominated by the smallest barrier, disfavoring other routes. Escapes from local minima occur preferentially through the neighbor with the smallest barrier instead of overcoming a typical barrier, $\beta_{\mathrm{loc}}=T_{\mathrm{loc}}^{-1}=\sqrt{2 \ln (M) / M}$, where $M$ is the number of states kinetically connected to a given state. In this work, the kinetic rules allow only a single

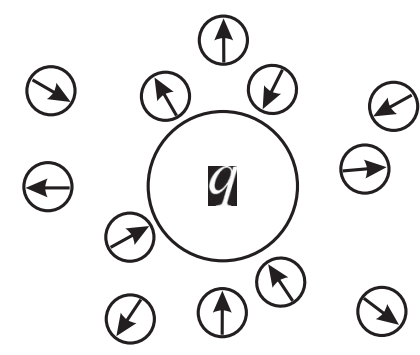

FIG. 2. Single-shell OW model for solvent dipoles around a cavity with charge $q$. dipole flip per elementary move, so $M=N$. In our simulations $N=20$, so $\beta_{\text {loc }}=0.54$. (b) Global phase transition is the one below temperature $1 / \beta_{g}$, where only a small set of low energy states are accessible. This is associated with the breakdown of the ergodicity. Also, the kinetics of the systems with different initial conditions, but with the same average energy distribution, can have different behavior. This is called non-self-averaging. Thermodynamically, this is the only phase transition; it is associated with the total number of states $(2 N)$, and the temperature is given by $\beta_{g}=T_{g}^{-1}=\sqrt{2 \ln (2)} \sim 1.17$ [9]. There is still another kinetic transition, the polarization-dependent phase transition [3]. It is associated with the distribution of energies at each polarization, but this transition is not addressed in this study.

Let us now turn our focus to the study of the kinetics and the associated statistical fluctuations of ET. In the Marcus description of ET, the reactions are assumed to occur when the system reaches the reactive region ( $x_{f}$ in Fig. 1). The dynamics of this system can be studied by the first passage time (FPT) of such events, $t$. FPT is the time the system diffuses from an initial total polarization $x_{i}$ to another final polarization $x_{f}$, where the ET occurs. The mean of FPT is denoted by $\tau$ (mean first passage time). A way to measure the significance of high order statistical fluctuations in kinetics beyond the mean level-the intermittency is to observe the ratio between the average of the $n$th order moment and the $n$th power of the first moment [7,1014], $R_{n}=\frac{\left\langle t^{n}\right\rangle}{\left\langle t t^{n}\right.}$, where $\langle t\rangle=\tau$. At high temperature the system is well behaved, and it is expected that the times of occurrence obey an exponential behavior with a single rate constant of reaction, $k=\tau^{-1}$. The times of occurrence (reaction) are distributed exponentially, so that the survival probability of events follows Poisson exponential kinetics [10], and $R_{n} / n !=1$. Below the transition temperatures, fluctuations on the FPT are expected to be significant and $R_{n} / n ! \gg 1$. The increase of this ratio indicates that the statistics is deviated from Poisson statistics. It implies that the high order moments become more and more important. Long tails of the distribution of FPT start to develop. Intermittency, where rare events play crucial roles in dynamics, is expected [7,10-14].

The kinetic rules of our simulations allow only a single dipole flip per elementary move, with move acceptance based on a Monte Carlo procedure [5,6]. A simulation with 20 dipoles was carried out with $\Delta E=20^{1 / 2}$ and $\bar{E}(x)=0$. In the simulations, the energy for the system is fixed according to the random energy model [3-6,9] for each of the $2^{N}$ states. Different runs correspond to varying the states of the dipole orientations $(+1$ or -1$)$. We investigate two different types of reactions controlled by the environments. The first one is the thermally activated reactions, illustrated in Fig. 1(a). After a thermal equilibration the system starts at $x_{i}=x_{\text {eq }}=0$ and diffuses to $x_{f}=0.5$. At temperatures around $T_{\text {loc }}$, local minima will be populated. 
As the temperature is lowered, $T_{\mathrm{loc}}$ will be the first transition temperature felt by the system. All FPTs were computed with the same energy assignment, but under different initial conditions. In Fig. 3 the distribution of FPT for well behaved single exponential decay is plotted along with the simulated populations for two temperatures. At high temperature, $\beta=0.3$, the single exponential fits well to the simulation data. At a temperature below the local transition temperature $(\beta=0.6)$, most of the data does not have large deviation from the exponential distribution. However, the parameter $R_{n} / n$ ! already indicates a great discrepancy with the values diverging from 1; see Table I. The kinetic simulation data at longer times $\left(t>10^{4}\right.$ steps $)$ show significant deviations from the exponential kinetics. Therefore, the ratio among the moments is a sensitive probe to the transitions and fluctuations in kinetics.

For the thermally activated reaction simulation [Fig. 1(a)], the reaction rate $(1 / \tau)$ as a function of inverse temperature $\beta=1 / T$ stays practically constant when the temperature of the system is high, until the temperature drops to approximately $\beta=0.4 .1 / \tau$ monotonically increases with the inverse of temperature, as shown in Fig. 3(b). Kinetics is significantly slower when temperature drops below $\beta=0.4$. In Fig. 3(c) shown $R_{n} / n$ ! is shown as a function of inverse temperature, where one can see the influence of the local temperature on the ratios among the moments $\left(R_{n} / n !\right)$, around $\beta^{\text {loc }}$. The Fig. 3(c) shows that the system, initially with low energy, corresponding to the polarization $x=0$, begins to get trapped around $\beta=$ 0.5. $R_{n} / n$ ! diverges dramatically at lower temperatures. The simulation has a time limit of $10^{9}$ Monte Carlo steps for FPT. At even lower temperatures, simulations are truncated and the system does not reach the final polarization in the allowed time.

We carried out another simulation corresponding to a relaxation process followed by ET reaction. It mimics experiments where the molecule in the cavity undergoes a dipole shift upon electronic excitation, which changes the equilibrium polarization, as shown in Fig. 1(b). The ET occurs after the relaxation, when the system is around the new equilibrium polarization. The system relaxes from high energy states and does not get easily trapped to local minima. The system was simulated initially at excited state leaving the initial polarization $x=-0.5$ until the final polarization $x=0.1$ [Fig. 1(b)]. Figure 4 indicates that the system becomes significantly trapped around global transition temperature $T_{g}$, and $R_{n} / n$ ! has its highest values close to $T_{g}$. As temperature is lowered even further $(\beta>$ $1.2), R_{n} / n$ ! decreases and tends to unity in this regime. The system has few accessible paths that the kinetics is governed by a dominant path escaping from a single deep trap. Fluctuations on $R_{n} / n$ ! at $\beta \sim \beta_{\text {loc }}$, however, occur in a less pronounced way.

We see that two characteristic temperatures control the nature of the kinetics. The global trapping temperature is
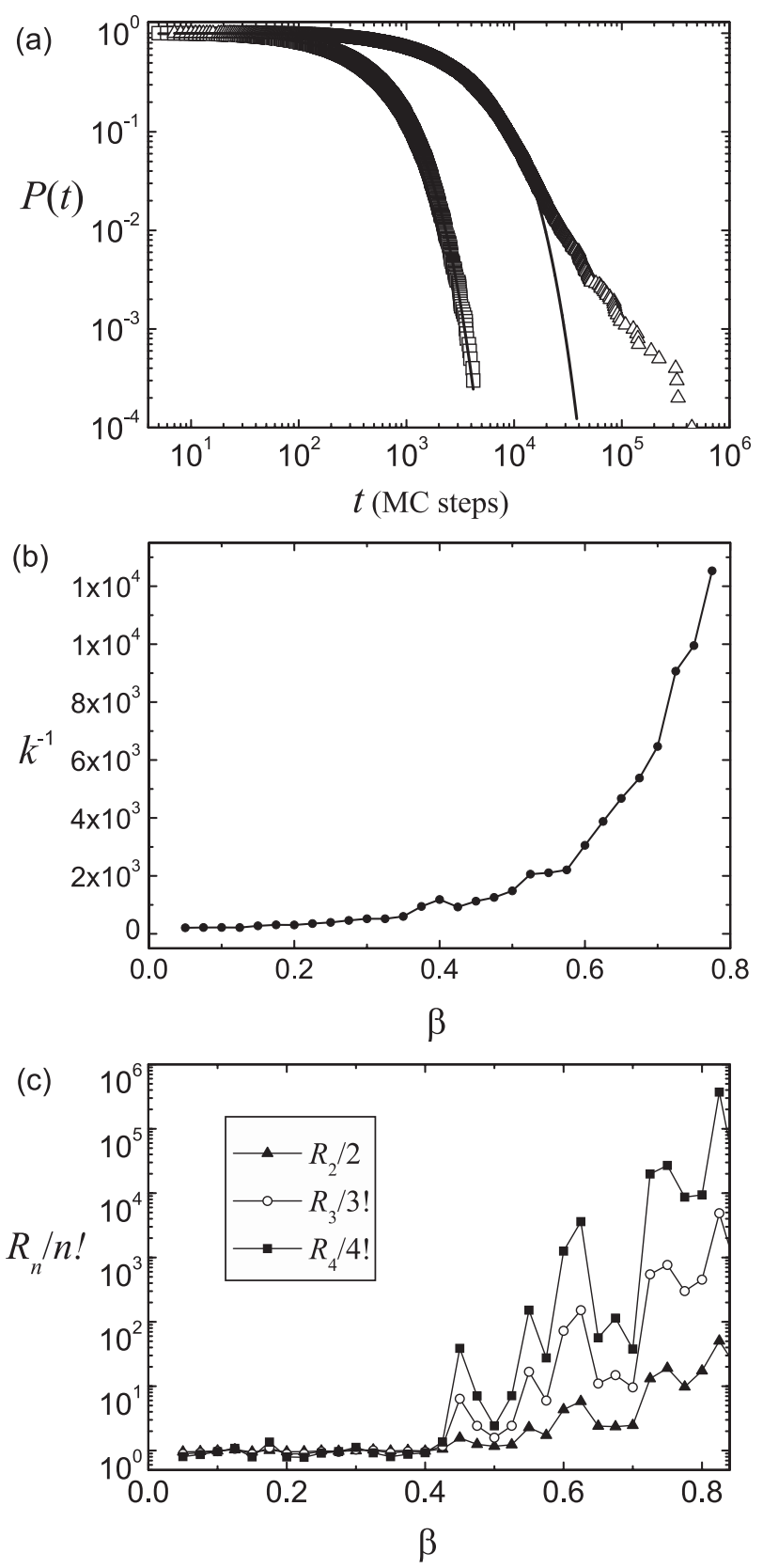

FIG. 3. Thermally activated process simulation (from equilibrium to activated state). (a) Comparison between the analytical and computational results, where $x_{i}=x_{\mathrm{eq}}=0$ and $x_{f}=0.5$. For $\beta=0.3(\square)$ the rate is $k=1.9 \times 10^{-3}$; for $\beta=0.6(\triangle)$ the rate is $k=3.2 \times 10^{-4}$. (b) Reaction rate as a function of inverse temperature. (c) $R_{n} / n$ ! as a function of inverse temperature, it deviates from 1 around the local transition temperature $\left(\beta_{\mathrm{loc}}=\right.$ $\left.T_{\text {loc }}^{-1}=0.54\right)$.

for the relaxation process. The local trapping temperature is for the activation process. Both parameters act as a marker where the kinetic process is single exponential at the temperature well above them, becomes nonexponential at the temperature around them, and is close to single exponential again at the temperature well below them. 
TABLE I. The values of $R_{n} / n$ !, found through simulation, for a high temperature and below the local critical temperature.

\begin{tabular}{cccc}
\hline \hline$\beta$ & $\mathbf{R}_{2} / \mathbf{2}$ & $\mathbf{R}_{3} / \mathbf{3 !}$ & $\mathbf{R}_{4} / \mathbf{4 !}$ \\
\hline 0.3 & 1.0 & 1.04 & 1.16 \\
0.6 & 3.34 & 52.2 & 1027.8 \\
\hline \hline
\end{tabular}

Similar kinetic behavior in biomolecular folding is observed [11-14]. We believe this exponential-nonexponential-exponential transition in temperature is a quite universal behavior in complex energy landscapes. At high temperatures, there are multiple routes to the final statesthe kinetics "senses" only the average barrier, resulting the single exponential kinetics. When the temperature is lowered, the local structures of the underlying landscape show up. When the states are nearly trapped or frozen to local minimum, the Gaussian distributed density of states can be linearized and expanded around the mean, resulting an exponential distribution of free energy. This will lead to the power law distribution of first passage time [11-14]. This kind of kinetic phenomena has been observed in single molecule electron transfer experiments [8]. Our model provides a microscopic origin for the fractional noise and power law distribution of kinetics due to the complexity of the underlying landscape. At the temperature well below the characteristic temperature, conformational space search is limited. The kinetics is dominated by the escape process from a single deep trap. Thus the kinetics is back to single exponential again. In the cryotemperature regime, there could still be a few traps or conformational discrete substates [15] around instead of a single one. Thus single exponential kinetics is hard to observe. Nevertheless, we should still be able to see the degree of the nonexponentialness become less pronounced

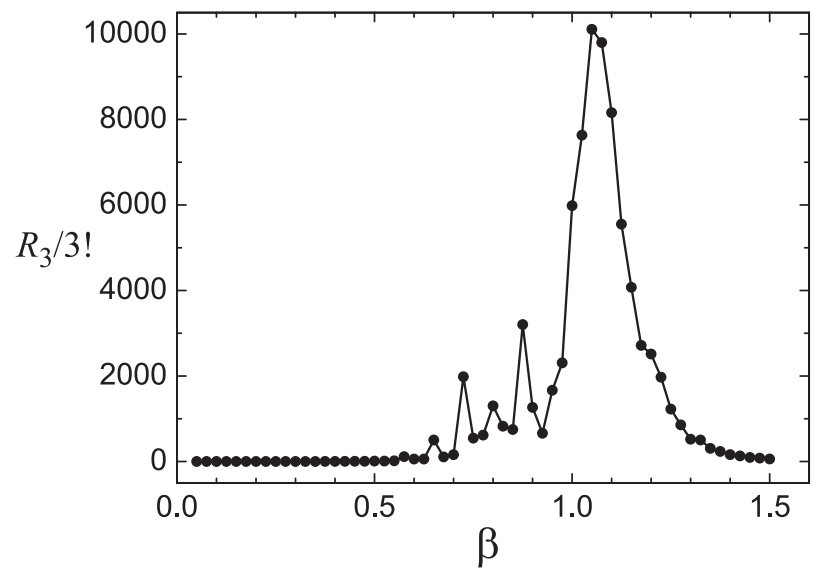

FIG. 4. $R_{n} / n$ ! for $n=3$ as a function of inverse temperature in the relaxation simulation $\left(T_{g}^{-1}=1.17\right)$, for $30 \mathrm{k}$ runs. For $n=2$ and 4 the results are qualitatively equivalent. as the temperature drops further in the low temperature regime in single molecule experiments.

In this work, we calculated the characteristic time associated with the corresponding reaction coordinate in ET. The relationship between this characteristic time and the full ET rate was studied [16] and illustrated for both the diffusional and transition state limits as well as for the general case.

We thank J.N. Onuchic and P.G. Wolynes for helpful discussions. V.B.P.L. and L.C.P. A. were supported by Fundação de Amparo à Pesquisa do Estado de São Paulo (FAPESP), Brazil. J. W. was supported by the NSF, ACS Petroleum Research Fund, and the K. C. Wond Foundation. M. N. was supported by DOE under Grant No. DE-AC0298CH10886.

*Electronic address: vleite@ibilce.unesp.br

${ }^{\dagger}$ Also at State Key Laboratory of Electroanalytical Chemistry, Changchun Institute of Applied Chemistry, Chinese Academy of Sciences, Changchun 130022, People's Republic of China.

Electronic address: jin.wang.1@ stonybrook.edu

[1] R. A. Marcus, J. Chem. Phys. 24, 966 (1956).

[2] P. J. Steinbach et al., Biochemistry 30, 3988 (1991); N. Agmon and J. J. Hopfield, J. Chem. Phys. 79, 2042 (1983); I. Rips and J. Jortner, J. Chem. Phys. 87, 2090 (1987); H. Sumi and R. Marcus, J. Chem. Phys. 84, 4272 (1986); W. Nadler and R. Marcus, J. Chem. Phys. 86, 3906 (1987).

[3] J. N. Onuchic and P. G. Wolynes, J. Chem. Phys. 98, 2218 (1993).

[4] Y. Tanimura, V. B. P. Leite, and J. N. Onuchic, J. Chem. Phys. 117, 2172 (2002).

[5] V. B. P. Leite and J. N. Onuchic, J. Phys. Chem. 100, 7680 (1996).

[6] V. B. P. Leite, J. Chem. Phys. 110, 10067 (1999).

[7] J. Wang and P. G. Wolynes, Phys. Rev. Lett. 74, 4317 (1995); J. Chem. Phys. 110, 4812 (1999).

[8] H. Yang et al., Science 302, 262 (2003); S.C. Kou and X.S. Xie, Phys. Rev. Lett. 93, 180603 (2004); W. Min et al., Phys. Rev. Lett. 94, 198302 (2005).

[9] B. Derrida, Phys. Rev. Lett. 45, 79 (1980); Phys. Rev. B 24, 2613 (1981).

[10] J. Wang, J. Chem. Phys. 118, 952 (2003).

[11] C. L. Lee, C. T. Lin, G. Stell, and J. Wang, Phys. Rev. E 67, 41905 (2003); J. Chem. Phys. 118, 959 (2003).

[12] Y. Zhou, Q. Zhang, G. Stell, and J. Wang, J. Am. Chem. Soc. 125, 6300 (2003).

[13] J. Wang, Biophys. J. 87, 2164 (2004).

[14] V.B.P. Leite, J. N. Onuchic, G. Stell, and J. Wang, Biophys. J. 87, 3633 (2004).

[15] H. Frauenfelder, F. Parak, and R. D. Young, Annu. Rev. Biophys. Biophys. Chem. 17, 451 (1988); H. Frauenfelder, S. G. Sligar, and P. G. Wolynes, Science 254, 1598 (1991).

[16] M.D. Newton and H.L. Friedman, J. Chem. Phys. 88, 4460 (1988); 89, 3400 (1988). 